\title{
Playgrounds: La Importancia Educativa del Espacio Exterior
}

\section{Playgrounds: The Educational Importance of Outdoor Spaces}

\section{Playgrounds: Importância Educacional do Espaço Exterior}

\author{
Virginia Navarro-Martínez * \\ Cuartocreciente arquitectura para la infancia
}

\begin{abstract}
El espacio exterior de juego (playground) en la infancia ofrece unas oportunidades de aprendizaje propias. Actualmente la educación se enfrenta a un cambio de paradigma. Este cambio implica replantearse los espacios donde la enseñanza tiene lugar y adaptarlo a los nuevos retos educativos. Y sin embargo, ¿es esto extensible a los espacios exteriores de juego? ¿Ha cambiado significativamente a lo largo del tiempo el modo en el que se juega? ¿A qué desafíos se enfrenta un espacio exterior destinado a la infancia en el s. XXI? Al mismo tiempo la arquitectura, volcada desde la actual crisis económica en su faceta social, se plantea por primera vez el alcance real que el diseño tiene sobre la vida humana. En este contexto los debates en foros internacionales plantean cuestiones como: ¿Puede el diseño desafiar la desigualdad? ¿Podemos “diseñar" la participación de la comunidad? ¿Puede el diseño recuperar el espacio público? ¿Cómo podemos diseñar en la escasez? En el necesario diálogo entre arquitectura y educación, el artículo enlazará ambas cuestiones con el objetivo de profundizar sobre el aprendizaje del niño en el espacio exterior. En un momento en el que el acceso libre del niño a estos espacios es cada vez más limitado, se abordarán los retos que éstos deben resolver y el alcance que el diseño puede tener en ellos.
\end{abstract}

Descriptores: Educación no-formal, Infancia, Juego, Arquitectura.

\begin{abstract}
The outdoor space for playing (playground) has its own learning opportunities. Nowadays education is about a paradigm shift. This change involves rethinking spaces where teaching takes place and setting new educational challenges. Nevertheless, is this applied to playgrounds? Has it deeply changed the way children play? What challenges must face an outdoor space for children and youngster in the twenty-first century? At the same time architecture, focused on social issues since the economical crisis, considers the real scope design has on human life for the first time. Discussion in international fora arise questions as: Can design challenge inequality? Can we design community engagement? Can design reclaim public space? How do we design with scarcity? Linking architecture and education, the article will delve further into children's learning in outdoor spaces. Nowadays children access to these spaces is becoming more and more restricted. That is the reason why it is important to tackle the problems current playgrounds must solve and the extent than design can have on them.
\end{abstract}

Keywords: Non-formal education, Childhood, Play, Architecture.

*Contacto: virginianavarro@telefonica.net

ISSN: 2254-3139

www.rinace.net/riejs/

revistas.uam.es/riejs
Recibido: $\quad 11$ de diciembre 2016

$1^{\text {a }}$ Evaluación: 7 de febrero 2017

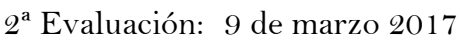

Aceptado: 11 de marzo 2017 


\begin{abstract}
$\mathrm{Na}$ infância o espaço exterior (playground) oferece oportunidades de aprendizagem específicas. Atualmente a educação enfrenta uma mudança de paradigma. Esta mudança obriga a repensar os espaços onde o ensino tem lugar além de implicar novos desafios educacionais. Isso, no entanto, será extensível aos espaços de jogo exteriores? A forma de jogar mudou significativamente ao longo do tempo? Quais são os desafios que enfrenta um espaço para as crianças no s. XXI? Ao mesmo tempo a arquitetura, afetada pela atual crise econômica no seu papel social, é considerada pela primeira vez em toda a extensão, através do projecto e da influencia que este tem sobre a vida humana. Neste contexto, as discussões nos fóruns internacionais levantar questões tais como: pode, o projecto, desafiar a desigualdade? podemos "projetar" a participação da comunidade? podemos projetar uma recuperação do espaço público? Como podemos projetar na escassez de meios? No diálogo necessário entre arquitetura e educação, o artigo irá analisar ambas as questões com o objectivo de aprofundar o tema da aprendizagem da criança no espaço exterior. Numa altura em que o acesso gratuito a estes espaços é cada vez mais limitado, serão abordados os desafios e o alcance que o projecto de arquitectura poderá ter.
\end{abstract}

Palavras-chave: Educação informal, Crianças, Jogo, Arquitectura.

\title{
1. La escasez del juego al aire libre
}

En 1560 Peter Brueghel el Viejo realizaba el cuadro titulado "Juego de niños". En él aparecen más de 230 niños realizando 83 juegos diferentes en un entorno urbano donde el adulto no existe. A pesar de los cinco siglos que lo separan de la actualidad, podemos reconocer en este lienzo bastantes juegos de nuestra propia infancia (y aún más si hablamos de nuestros padres o abuelos) e incluso algunos que todavía permanecen en ciertas plazas públicas y patios escolares. De hecho no es el tipo de juego en el espacio exterior lo que se ha visto profundamente transformado a lo largo del tiempo, podemos afirmar que la actitud lúdica de los niños del cuadro permanece inalterada, sino la posibilidad de jugar en un contexto libre y sin supervisión. Por primera vez en toda la historia de la infancia nos enfrentamos a una limitación radical del uso del espacio exterior ${ }^{1}$. Por ello es urgente determinar en qué medida afecta esta pérdida al desarrollo infantil y de qué forma el diseño y determinadas estrategias espaciales, sociales y educativas pueden contribuir a su mejora.

Al mismo tiempo se gestan nuevos modelos de enseñanza que buscan responder a las necesidades formativas actuales, replanteándose esta revisión los espacios que deben darles cabida. Si bien parece que el modelo tradicional del aula está a punto de ser trascendido, ¿qué sucede con el diseño del espacio exterior? ¿Está siendo considerado? ¿No deberíamos pensarlo desde las mismas pautas ya que se trata de un espacio educativo?

\section{La importancia educativa del espacio exterior}

El espacio exterior es imprescindible en la infancia. De forma genérica en él se realizan actividades de psicomotricidad gruesa, juego libre y exploración espontánea. El acceso frecuente al mismo, siguiendo la enumeración anterior, ya permite anticipar algunos beneficios: una mejora de la salud (la actividad vigorosa de los niños, estimada de forma

\footnotetext{
${ }^{1}$ Es interesante en este sentido todo el trabajo desarrollado por el grupo de trabajo La Ciudad de los niños perteneciente a la asociación Acción Educativa. Bianualmente realizan encuentros posteriormente condensados en una publicación en los que aparece recurrentemente analizado este tema.
} 
óptima en noventa minutos, está actualmente reducida a veinte), un incremento de la empatía, un desarrollo adecuado del aprendizaje social y una mejora del juego imaginativo.

Pero el espacio exterior también aporta otras experiencias insustituibles para el desarrollo cognitivo: el contacto con seres vivos como animales y plantas, la observación de fenómenos naturales (lluvia, viento, sol, etc.), el juego con materiales moldeables (arena, agua) y el aprendizaje de la relaciones de dependencia entre el sistema social y el sistema natural. Una de las enseñanzas más importantes del espacio exterior es el proceso de socialización, ya que en él se interacciona libremente con otros niños y adultos y se adquieren habilidades como el respeto y la colaboración. También, con su dosis de exploración, favorece la denominada pedagogía del asombro enunciada por Hannoun.

\section{Contextos del espacio exterior en la infancia: Distintos entornos, distintos aprendizajes}

Si nos centramos en el contexto de la infancia, podemos distinguir tres entornos principales en los que ésta se relaciona con el espacio exterior: el patio escolar, la ciudad (o medio construido) y el entorno natural. Profundizaremos en este artículo en las características principales de los dos primeros.

En el primer caso (el patio escolar) las actividades al aire libre van asociadas al concepto de recreo o deporte, momentos dedicados al descanso y al ejercicio físico como actividades complementarias al aprendizaje del aula. Fue a finales del s. XIX, con el triunfo de la escuela pública, obligatoria y laica, cuando se instituyó el recreo de manera generalizada con la finalidad de reponer a los alumnos de la fatiga ${ }^{2}$. Desde entonces la actividad al aire libre es la otra cara de la moneda de la educación formal, ya que se ha demostrado que estos periodos de esparcimiento benefician al aprendizaje. Como ejemplo de la vigencia y alcance de esta idea destacamos el proyecto The playtime project que actualmente (2017) la asociación London Play está desarrollando como experiencia piloto en algunos colegios de Londres. ${ }^{3}$ Su objetivo es demostrar que los niños aprenden mejor cuando tienen oportunidades regulares de juego. Este concepto se inspira en el sistema educativo finlandés para primaria, donde el juego se considera una herramienta de aprendizaje y la actividad predominante que deben realizar los niños de esta edad. De hecho las escuelas de este país poseen recreos de 10-15 minutos entre cada una de sus lecciones.

Pero el equilibrio mental y físico del niño no sólo se ve beneficiado cuando éste juega, sino que el mero paseo a pie o en bici por la ciudad produce mejoras en el estado de ánimo, la concentración, la creatividad y la habilidad para resolver problemas. Estas ideas, junto con la necesaria recuperación de la autonomía urbana del niño, son la base científica sobre las que se asienta la propuesta de caminos escolares (recorridos urbanos seguros para que el niño realice solo el camino que separa su casa de la escuela).

A estas ideas hay que superpone el problema de la pérdida del juego al aire libre fuera del ámbito escolar. De esta forma el patio de recreo ha adquirido un peso específico y una responsabilidad mayor que en cualquier otro momento de su historia. La falta de

\footnotetext{
${ }^{2}$ Recuperado de http://www.me.gov.ar/monitor/nro10/museo.htm [Consulta 14/01/2017]

3 Recuperado de http://www.childinthecity.eu/2016/10/12/putting-play-at-the-heart-of-londons-schools/ [Consulta 14/01/2017]
} 
consideración del niño en el diseño del espacio urbano, la prioridad del coche frente al peatón, la sobreprotección por parte de los padres, el exceso de actividades extraescolares y deberes y la introducción de nuevas tecnologías han finalizado por convertir estas actividades en algo minoritario y en ocasiones inexistente en la jornada infantil. Así que el patio hoy día no sólo complementa y favorece la educación, sino que se ha convertido en ocasiones en el único espacio en el que el niño puede establecer relaciones e interactuar con otros niños de forma continuada.

Por último también son remarcables las oportunidades de aprendizaje que el espacio exterior ofrece en sí mismo y no como complemento a la educación formal, incrementadas cuando en ellos existen elementos naturales. De hecho el uso del jardín como parte fundamental de la educación del niño ya fue introducida por Froebel en 1840 en sus Kindergarten en Alemania. A finales del XIX los jardines se incorporaron en las escuelas de Europa y Norteamérica para dotar a los niños de habilidades vitales básicas, así como introducirlos en los beneficios económicos de la agricultura. En Suecia, Austria, Alemania, Bélgica y Rusia los jardines fueron obligatorios en las escuelas, mientras que en Inglaterra los salarios de los profesores dependían del nivel productivo de sus cultivos. En 1930, los avances tecnológicos en agricultura, el desarrollo de los supermercados y el currículum orientado a test y exámenes hizo que se abandonaran. No obstante, estamos siendo testigos de una vuelta de la jardinería y al contacto con la naturaleza en las escuelas, gracias en parte a las aportaciones de autores como Richard Louv. Louv ha investigado la relación de los niños con el mundo natural desde una perspectiva histórica hasta llegar a nuestros días y ha creado el término nature-deficit disorder para describir las consecuencias negativas que tiene en la salud y las relaciones sociales del niño la carencia de contacto físico con el mundo natural. De hecho la búsqueda de esta relación se ha convertido en el motor de algunas escuelas infantiles europeas y americanas, ubicadas en medio de bosques, entre las que destacan las Waldkindergarten ("escuela infantil del bosque") en Alemania, las I Uro ch Skur ("vamos fuera llueva o haga sol") en Suecia o las Skov Skole ("colegio del bosque") en Dinamarca4. La naturaleza ofrece maravillosas oportunidades de aprendizaje, incluso si su inclusión en la escuela es sólo a través del jardín. No sólo en el sentido ecológico, sino que su estudio puede combinarse con el arte, la lectura, la escritura, la ciencia e incluso con los estudios sociales.

El segundo espacio que abordamos (la ciudad) está íntimamente relacionado, como ya hemos ido avanzando, con el patio escolar. A diferencia del juego limitado en el tiempo y entre iguales que tiene lugar en el recreo, la ciudad ofrece una interacción más rica con el medio a nivel físico (mayor número de estímulos), social (amplio rango de edades y personas), cultural (inserción en un medio determinado) y temporal (permite un desarrollo del juego más dilatado).

La creación de espacios urbanos específicamente destinados a la infancia (playgrounds) surgen asociados a los cambios sociales que tuvieron lugar a finales del XIX, coincidiendo con el momento en el que se incorpora el recreo en las escuelas. Estas primeras áreas de juego estaban vinculadas a los parques públicos con el objetivo de solventar la necesidad de jugar al aire libre de los más pequeños y protegerlos del peligro de las calles. En los años 50 y 60, con el baby-boom y la generalización del uso del automóvil, dichos espacios fueron adquiriendo importancia de forma proporcional al aumento del tráfico en las calles.

\footnotetext{
${ }^{4}$ Recuperado de http://bosquescuela.com/experiencias-internacionales/a [Consulta: 14/01/2017]
} 
Su diseño ha estado a menudo relacionado con los avances pedagógicos de los patios escolares (los areneros que aún hoy aparecen en numerosos parques infantiles tienen su origen el Froebel) y actualmente cualquier equipamiento infantil industrializado fundamenta su razón de ser principalmente en principios pedagógicos y de seguridad.

No obstante, el principal espacio urbano que el niño ha perdido es la calle. Francesco Tonucci reclama en su libro "La ciudad de los niños" la necesidad de que la ciudad vuelva a incorporar en sus aceras y plazas a la infancia y Jane Jacobs ya expuso en los años 60 las oportunidades que este espacio brindaba. La calle es para esta urbanista un lugar más seguro que los parques públicos porque en ella toda la comunidad adulta se responsabiliza de los más pequeños "mientras se dedican a otras cosas". También cuenta ejemplarmente el aprendizaje cívico que esto supone:

En la vida real, los niños sólo pueden aprender de la vida en común de los adultos en
las aceras de la ciudad (si es que lo aprenden) el principio más fundamental de una
buena vida urbana: todo el mundo ha de aceptar un canon de responsabilidad pública
mínima y reciproca, aún en el caso de que ningún vínculo afectivo les una. [...] La
lección de que los residentes de una ciudad han de aceptar una cierta responsabilidad
de lo que ocurre en la calle la aprenden una y otra vez los niños de las aceras que
disfrutan de una vida pública local. Demostrarán haberla asimilado si dan por
sentado, al cabo de un tiempo, que también ellos son parte de la plantilla.

También advierte que parte del encanto de las actividades que se realizan en la calle (escribir con tiza, saltar a la cuerda, patinar, decorar cajas, etc.) está en la complementaria sensación de libertad que conlleva, algo que no sucede cuando éstas deben hacerse en un lugar oficialmente destinado a ello. Según la autora estas acciones deben ser espontáneas y encontrar espacios inmediatamente accesibles, anunciando su desaparición (tal y como ha sucedido) si tales condiciones se pierden.

Reforzando la lectura pedagógica del juego en la ciudad, también conviene destacar las reflexiones de la maestra Penny Ritscher. En 2006 elaboró una lista de los valores educativos que conllevaba esta actividad:

- Se jugaba fuera de casa, es decir, fuera del control directo de los adultos. Los niños aprendían a organizarse entre sí.

- Los más mayores se hacían responsables de los más pequeños.

- Como se tenían pocas cosas, éstas se sabían aprovechar.

- Se sabía sacar provecho de los materiales ocasionales (piedrecitas, cáscaras, hierba, tierra). Al jugar con materiales pobres, se utilizaba y se desarrollaba la creatividad.

- Eran juegos con un fuerte componente sensitivo (olor a hierba, tierra mojada, etc.).

- Se tomaba posesión del espacio: se conocía el territorio.

- Los peligros eran asumibles. Se aprendía a medir las propias fuerzas ente los riesgos.

- Se tomaba posesión del tiempo. Los tiempos eran largos pero "nunca había bastante tiempo".

- Había continuidad en los juegos. Los niños sabían hacerlos durar por capítulos.

- Eran experiencias compartidas. 
Hoy en día los parques infantiles constituidos por una serie de mobiliario de juego prefabricado, en los que la seguridad física prima sobre el estímulo, representan todo el espacio que desde el planeamiento urbanístico se destina a la infancia. La inexistencia por tanto de espacios públicos ricos en experiencias, la desaparición de la vida en la calle, la falta de autonomía y de tiempo del niño y la aparición de juegos alternativos basados en las nuevas tecnologías han concluido por finalizar con la presencia de la infancia en las calles.

Con las premisas anteriormente expuestas empezaremos a analizar los desafíos que debe afrontar hoy día un espacio exterior de juego.

\section{Desafíos actuales y nuevas oportunidades educativas}

Loris Malaguzzi hablaba del espacio como el tercer profesor del niño. Quizás son los espacios exteriores de la infancia, por ser escenarios de una educación no formal, uno de los lugares donde este concepto adquiere especial relevancia. Prueba de ello es la aparición en 1990 de las ciudades educadoras, donde se trata de articular y promover unos principios básicos para el impulso educativo de la ciudad 5 , entendiendo que el entorno es determinante para la formación integral de cada persona y capaz de promover actividades de plena igualdad que favorecen el hecho de sentirse respetado y ser respetuoso. Reconocer el potencial educativo del espacio urbano supone un gran avance a la hora de intervenir en su diseño. En la carta que recoge sus principios cabe destacar el reconocimiento de niños y jóvenes como ciudadanos de pleno derecho, con posibilidades de asociarse y participar según su grado de madurez.

Con esta visión educativa y el análisis anteriormente abordado podemos enumerar los principales desafíos que afronta actualmente el espacio exterior. Para ello distinguiremos entre el entorno escolar y urbano:

El patio escolar:

- Debe enriquecer la oferta de juegos por edades. Actualmente esta oferta es pobre o prácticamente inexistente.

- Debe prevenir la desigualdad (de género, social o cultural).

- Debe ser accesible a todos (considerar las barreras arquitectónicas).

- Debe relacionarse con su contexto. El patio puede ser fuera del horario escolar un espacio público para los niños o la comunidad.

- Debe incluir elementos naturales.

- Debe permitir la transformación por parte de los alumnos.

- Debe favorecer los retos físicos y cognitivos.

- Debe poseer un espacio suficiente para cada alumno. A menudo las ratios de espacio libre por niño son insuficientes, incrementando la agresividad.

${ }^{5}$ Es interesante la lectura completa de la carta. Recuperado de http://www.edcities.org/carta-de-ciudades-educadoras/ [Consulta 14/01/2017] 
- Debe revisar el tiempo destinado a su uso (interacción más frecuente) y entenderse como un lugar de trabajo, observación, juego y actividades variadas.

- Debe relacionarse de forma más fluida con el aula (desde visual hasta conceptualmente).

Como cierre de esta enumeración, y a medio camino entre el espacio escolar y urbano, habría que añadir el desafío planteado por los caminos escolares: que los niños puedan realizar autónomamente el recorrido entre la escuela y sus casas.

Los espacios urbanos:

- Deben dar prioridad al peatón frente al coche.

- Deben favorecer el intercambio intergeneracional.

- Deben promover el respeto y la justicia social.

- Deben integrar la diversidad cultural.

- Debe prevenir la desigualdad (de género, social o cultural).

- Debe ser accesible a todos (considerar las barreras arquitectónicas).

- Deben ser seguros, implicando para ello a la comunidad.

- Deben recoger las necesidades específicas de ocio de niños y adolescentes.

- Deben integrar la naturaleza en la medida que esto sea posible y ser confortables.

- Deben estar vinculados a su contexto.

- Deben ofrecer desafíos físicos y cognitivos. La seguridad es importante pero no debe primar sobre otros valores educativos.

- No se debe reducir el uso de la ciudad por la infancia a pequeños espacios destinados a un único rango de edad que repiten un catálogo de mobiliario urbano.

- Deben favorecer que su diseño se haga desde la participación incorporando la visión de niños y adolescentes.

Éste último apartado, aplicable también al espacio escolar y recogido en los fundamentos de las ciudades educadoras, representa quizás la oportunidad de aprendizaje más novedosa. Los niños por primera vez pueden decidir y modificar su propio entorno de forma democrática, aplicando así plenamente el artículo 31 de la Convención de los derechos del niño en el que se reconoce tanto su derecho al juego y al esparcimiento como el derecho a la participación en aquellas decisiones que les afecten ${ }^{6}$. De hecho el auge actual de la educación en arquitectura está íntimamente relacionado con la necesidad formativa de los niños acerca de su entorno construido. Los procesos participativos en la infancia demandan la necesidad de este tipo de enseñanza con el objetivo de desarrollar su capacidad crítica, entender y aplicar conceptos como sostenibilidad o ecología y obtener las herramientas necesarias para mostrar sus propias ideas.

Por su parte Liane Lefaivre, especialista en los espacios de juego holandeses realizados por el arquitecto Aldo Van Eyck, analiza la otra cara de la moneda y recoge el interés que aporta a la ciudad aquellas lecturas que incorporan a los niños en el diseño urbano. Éstas

${ }^{6}$ Convención de los derechos del niño. Recuperado de http://noticias.juridicas.com/base_datos/Admin/ircdn.html [Consulta 14/01/2017] 
nos remiten a una forma concreta de urbanismo en el que prima la pequeña escala, se valora el cuidado de los espacios cotidianos y se busca la calidad de vida a nivel de calle. Su visión urbana plantea no sólo la necesidad de volver a considerar seriamente al niño dentro del urbanismo, sino de valorar el potencial que los espacios dedicados a la infancia tienen como lugar público y de relación comunitaria dentro del tejido urbano.

Por último y de forma general, los espacios exteriores destinados a niños y adolescentes deben considerar la incidencia que en ellos tienen los factores sociales, anteriormente expuestos, que afectan a su uso: la sobreprotección de los padres, la falta de autonomía y la escasez de tiempo (debido a las actividades extraescolares o deberes), la deriva del juego hacia las nuevas tecnologías o la pérdida de una comunidad urbana interrelacionada.

\section{Aportaciones desde el diseño}

Actualmente la arquitectura se encuentra inmersa en su faceta más social. Tras la crisis económica de 2008 el descenso de la actividad en el campo de la construcción ha dado lugar a una preocupación por el espacio vacío así como por la reutilización de lo existente, recuperándose con renovado interés el espacio público de la ciudad. Además el arquitecto ha pasado de ser el autor de obras emblemáticas a mediador en el diseño de los vacíos urbanos, incorporando en su diseño no sólo la opinión de los ciudadanos sino incluso la mano de obra voluntaria de los mismos.

En este contexto surgen temas de debate como los planteados por el jurado del Curry Stone Design ${ }^{7}$, un galardón entregado a proyectos de carácter social cuyo alcance ha sido especialmente reseñable. Algunas de las preguntas planteadas para este año (2017): ¿Puede el diseño desafiar la desigualdad? ¿Podemos "diseñar" la participación de la comunidad? ¿Puede el diseño recuperar el espacio público? ¿Cómo podemos diseñar en la escasez? En general los diferentes foros tratan de determinar la capacidad social del diseño, la forma de integrar adecuadamente los procesos participativos y algunas soluciones desde la que intervenir con pocos medios.

Es interesante trasladar estas cuestiones al problema planteado en el artículo. Como puede anticiparse, hay cuestiones sociales que posiblemente requerirán más tiempo, pero está claro que el diseño puede favorecer unas respecto a otras. Como punto de partida, podemos enunciar dos: a) Influencias directas o aquellas que provocan, impiden, facilitan o dificultan una determinada conducta o proceso de desarrollo. Un ejemplo de este tipo de influencia puede verse cuando introducimos una estructura de tubos en el patio escolar y, en poco tiempo, la conducta de trepar se ve sensiblemente incrementada. b) Influencias indirectas. Cuando no es el ambiente en sí mismo, sino la percepción e interpretación que los sujetos hacen de distintos aspectos del ambiente lo que condiciona su conducta y la marcha de las actividades. Esto puede observarse en recintos extremadamente limpios que, aunque no esté indicado explícitamente, nos están comunicando que no se puede arrojar nada al suelo $^{8}$.

\footnotetext{
7 Aquí aparecen sólo algunas de las preguntas que se debatirán a lo largo de 2017 en el Curry Stone Design Prize. Recuperado de http://currystonedesignprize.com/ [Consulta 14/01/2017]

8 Cita extraida del manual Algo más que un patio de recreo, pág. $32 . \quad$ Recuperado de http://www.juntadeandalucia.es/medioambiente/consolidado/publicacionesdigitales/40380_ALGO_MAS_QUE_UN_PATIO_DE_RECREO/4O-

380/4_CRITERIOS_Y_REURSOS_METODOLOGICOS.PDF [Consulta 14/01/2017]
} 
Sin embargo, para exponer la influencia que tiene el diseño en la vida del niño y la comunidad no recurriremos a un listado, sino a tres modelos concretos que resultan ejemplares: el parque de aventuras Glamis (Reino Unido), Superkilen park (Dinamarca) y Geopark (Noruega)

El parque de aventuras Glamis se sitúa en una zona deprimida del este de Londres en la que existen grandes tasas de desempleo y un índice de pobreza infantil en torno al $60 \%$. Originalmente ocupó el vacío que había dejado un hospital demolido, siendo uno de los primeros parques de aventuras londinenses (1969). Los parques de aventuras son áreas de juego donde los niños pueden jugar con material de desecho para hacer sus propias construcciones y columpios (disponiendo para ello de herramientas y la ayuda de un adulto), hacer fogatas, utilizar coches en desuso y cualquier otra actividad que implique transformar el espacio. Desde el punto de vista didáctico, a pesar de lo alejado que está hoy día de los estándares de seguridad, es enormemente formativo y fue muy exitoso en los países nórdicos, Alemania, Suiza e Inglaterra, conservándose aún activos algunos de ellos. En el caso del Glamis Adventure Playground este gran espacio de juego ha servido para generar un lugar aglutinante de toda la comunidad circundante (de diversas orígenes) con el que han podido identificarse. A pesar de que se ha visto obligado a cerrar en distintas ocasiones, el recuerdo de los que jugaron en él con anterioridad ha impulsado siempre su permanencia desde la colaboración y la participación llegando hasta nuestros días. Su ejemplaridad como espacio de juego le ha hecho ser galardonado con distintos premios. En el conviven los espacios de construcción (niños entre 6-16 años) con un taller para reparar las bicicletas, huertos urbanos, una cafetería de comida saludable y una serie de actividades específicas para personas con discapacidad. Invitan además a sus instalaciones a músicos locales, grupos de teatro y gente del circo para que los que los niños (y adultos) jueguen ${ }^{9}$.

El segundo ejemplo es el Superkilen Park en Copenhague. Este parque de más de 800 metros de largo se inserta en el corazón del barrio más diverso y complejo (étnica y socialmente) de la ciudad. Se trata de un proyecto que mezcla arquitectura, paisajismo y arte. En su recorrido se intercalan una colección heterogénea de objetos procedentes de cada una de las nacionalidades que conviven en el barrio (60 en total). Se trata de objetos de uso cotidiano que, sacados de contexto, se convierten en elementos de juego. Cada uno de ellos incorpora una leyenda en danés y en el idioma de la ciudad de la que procede. La finalidad es reproducir un enclave urbano con la idea de jardín universal. Superkilen es un espacio pensado para los vecinos, que de manera indirecta participaron en todo el proceso de diseño. Al igual que en el caso anterior, ha logrado algunos retos difíciles: crear un lugar multicultural en el que sentirse integrado, transmitir la idea de respeto e igualdad y conseguir identificar a sus habitantes con la propuesta urbana ${ }^{10}$.

El último ejemplo es Geopark, ubicado en Stavanger (Noruega). Este parque presenta un conjunto de características muy interesantes que trascienden el espacio urbano convencional infantil o juvenil. Stavanger es una ciudad destacada en el país por ser el centro más importante de la producción petrolífera. Cuando se solicitó un parque infantil junto al Museo Noruego del Petróleo, los diseñadores decidieron contar para el mismo

\footnotetext{
${ }^{9}$ Recuperado de http://www.shadwellcommunityproject.org/wordpress/ [Consulta 14/01/2017]

10 Recuperado de http://www.experimenta.es/noticias/arquitectura/superkilen-parque-urbano-en-copenhague-de-big$3819 /$ [Consulta 14/01/2017]
} 
con tres recursos específicos de la zona: por una parte con los expertos geográficos y sísmicos de la industria del petróleo, en segundo lugar con el material (incluido de desecho) vinculado a su producción y finalmente con la intervención de grupos de jóvenes locales para la programación de las actividades del nuevo parque. El parque reproduce en su topografía la reserva de gas y petróleo más importante de Noruega y muestra de forma colorista las capas geológicas que se encuentran sumergidas a más de 2000 metros bajo su principal plataforma petrolífera. Tras esta primera definición topográfica, la segunda fase implicó a los jóvenes en la decisión de los usos entre los que propusieron paseo de bicicletas, escalada, espacio expositivo, zona de conciertos, zona de saltos, juegos de pelota, pista de skate, zonas de graffiti y áreas de descanso. En la tercera fase las superficies e instalaciones se crearon usando, reciclando y reformando elementos de las plataformas petrolíferas. El parque actualmente es usado por niños, padres y jóvenes a todas horas ${ }^{11}$.

Los tres ejemplos anteriores sirven para demostrar que el diseño puede ser clave en aspectos tan difíciles como la integración intercultural, la transmisión de actitudes de respeto e igualdad, la identificación e implicación de los vecinos en su gestión y mantenimiento, la incorporación de un amplio rango de edad e incluso contar a través de sus elementos, como en el último caso, la historia del lugar donde se ubica. Este potencial educativo del diseño debería ser tenido en cuenta cuando se proyectan espacios públicos, especialmente si van destinados a la infancia. Este artículo trata de demostrar la evidencia de que las cualidades de los espacios exteriores escolares o urbanos resulta hoy día insuficiente y que son enormemente importantes para un momento donde los desafíos a nivel social son tan relevantes.

Evidentemente estos avances deben ir acompañados de otras estrategias sociales, políticas y familiares para que resulten plenamente eficaces. Pero a la vista de la transcendencia formativa que su uso conlleva deberíamos desde el momento presente manifestar y favorecer su adaptación a las nuevas necesidades a las que se enfrentan hoy día niños y jóvenes.

\section{Referencias}

Cabanellas, I. y Eslava, C. (2005). Territorios de la infancia: Diálogos entre arquitectura y pedagogía. Barcelona: Graó.

Herrington, S., Lesmeister, C., Nicholls, J. y Stefiuk, K. (2007). An informational guide to young children's outdoor play spaces. Vancouver: Westcoast Childcare Resource Centre.

Jacobs, J. (2011). Muerte y vida de las grandes ciudades. Madrid: Capitán Swing Libros.

Lefaivre, L. (2007). Ground up cities. Play as a design tool. Rotterdam: 010 Publishers.

Martínez Soriano, J. P. y Valera Portillo, L. (1992). Algo más que un patio de recreo. Del patio de recreo a la recreación del medio. Sevilla: Junta de Andalucía.

Ritscher, P. (2006). El jardín de los secretos. Barcelona: Octaedro.

Tonucci, F. (1997). La ciudad de los niños. Madrid: Fundación Germán Sánchez Ruipérez.

\footnotetext{
${ }^{11}$ Recuperado de http://www.helenhard.no/projects/geopark/ [Consulta 14/01/2017]
} 


\section{Breve CV de la autora}

\section{Virginia Navarro-Martínez}

Es arquitecta por la ETSA de Sevilla (2003) y Máster en Arquitectura y Patrimonio Histórico (2008). Primer premio por su fin de carrera en la XXI Edición del Premio Dragados. Se forma en el estudio de Ricardo Alario, con quien comparte actualmente actividad profesional. En 2011 funda junto a Tibisay Cañas, Laura Organvídez, Ana Parejo y Sara Parrilla Cuartocreciente arquitectura, una iniciativa creada con el objetivo de mejorar los tres espacios principales en los que se desarrolla la niñez (casa, escuela y ciudad) a través de la investigación, los talleres de arquitectura, la realización de proyectos y el diseño de objetos. Actualmente desarrolla un tesis sobre el espacio de juego exterior en la infancia, dirigida por Ángel Martínez García-Posada. Ha escrito y presentado diversas comunicaciones sobre el playground y el juego del niño en la ciudad. Ha sido coordinadora junto a Jorge Raedó del III Encuentro Internacional de Educación en Arquitectura para la infancia y la juventud celebrado en enero de 2016 en el Museo Nacional Centro de Arte Reina Sofía (Madrid) y actualmente coordina y aporta contenido teórico a Ludantia: I Bienal Internacional de Educación en Arquitectura para la infancia y la juventud dirigida por Jorge Raedó y Xosé Manuel Rosales. ORCID ID: 0000-0002-6801-6653. Email: virginianavarro@telefonica.net 